\title{
Effect of Isotretinion on Rat's Liver and Aptness of Cumin Oil in Protection
}

\author{
Prshang Tawfiq (MSc) ${ }^{1}$, Tuqa Yousif Sharef(MSc) ${ }^{2}$, Khabat Anwar Ali $(\mathrm{PhD})^{3}$ and
} Lana Sardar Salih $(\mathrm{PhD})^{4}$

\section{Abstract}

Background: Isotretinoin is a vitamin derivative also known as 13-cis-retinoic acid used in the treatment of severe acne and some forms of skin, head and neck cancer. Some studies have shown that isotretinoin induces apoptosis in various cells in the body. Isotretinion has not been clearly implicated in cases of clinically apparent acute liver injury with jaundice. The mechanism by which isotretinoin causes serum aminotransferase elevations is not known, but it may represent a direct toxic effect, in that it appears to be more frequent with higher dose therapy. For prevention or healing natural herb and its oil are used. The Cumin oil is one of these herbal oils which used as antioxidant and anti-inflammatory.

Objective: To evaluate the histological damage of liver after treatment with the Isotretinoin and find out if cumin oil which was anti-inflammatory natural herb can protect liver from damage.

Patients and Methods: 30 male wistar rats weighing 200-250g were used in this study divided into five groups: the first group was control, second was treatment group (T1), receive $10 \mathrm{mg}$ of Isotretinion; the third group was (T2) receives $20 \mathrm{mg}$ of Isotretinion. And last two others were cumin oil groups including (T3) receive $10 \mathrm{mg}$ of Isotretinion plus Cumin oil and (T4) receive $20 \mathrm{mg}$ of Isotretinion plus Cumin oil. The experiment was accomplished in three weeks. In the end of each week, animals from each group were killed and dissect and liver taken for histopathological study. Blood collected for liver functional enzymes evaluation. Liver functional enzymes include alkaline phosphatase, S-GOT, S-GPT, T.S.B, Bilirubin direct and Bilirubin indirect.

Results: The study results show a significant difference between the control groups and treatment (T1) group in the values of alkaline phosphatase. A significant difference also found in the result of serum GOT between the T2, T3and T4 groups. The T3 group shows the highest significance between the other groups. The result of GPT shows no significant differences between the study groups except $\mathrm{T} 3$ group which show significant $\mathrm{P}<0.05$. The data of total serum bile (T.S.B) and indirect bilirubin test shows no significant differences among the study groups while analyzing data of direct bilirubin shows significant differences in T1and T2 comparing to other study groups graph. The histological examination of the rat's liver shows variable changes in the treatment groups during different time of the study. Isotretinion affect the texture of liver tissue mains noticed alteration includes infiltration of inflammatory cell around central vein, congestion of sinusoid and vacuolar degeneration in 
hepatocytes the treated groups with cumin oil (T3 and T4) shows a type of tissue protection comparing to other groups (T1and T2).

Conclusion: The present study conclude that long exposure to isotretinion cause hepatic damage, alteration in the liver texture and affect the functional liver enzyme level specially with high dose. Also the study concludes that the cumin oil is efficient in facing the toxicity effect of isotretinion and reverses the effect.

Keywords: Isotretinion, 13-cis-retinoic acid, Cumin oil, liver damage.

Corresponding Author: tuqa_yusef@yahoo.com

Received: $12^{\text {th }}$ September 2019

Accepted: $22^{\text {th }}$ October 2019

DOI:https://doi.org/10.26505/DJM.18024920912

\footnotetext{
${ }^{1,2}$ Department of basic science-Dentistry College - Hawler Medical University - Irbil -Iraq

${ }^{3}$ Collage of Education - Salahaddin University -Irbil-Iraq

${ }^{4}$ College of Science - Salahaddin University -Irbil-Iraq
}

\section{Introduction}

Looking for a beauty nowadays become obsession for many people. Beauty cannot be defined by age, gender, color, body shape or size , every one search for beauty specially teenage and young ones. Isotretinoin which is a vitamin A derivative (also known as 13-cisretinoic acid) is one of the important ingredients used in cosmetics, hair and skin care products. Isotretinion is recommended for the treatment of severe inflammatory acne of the nodulocystic types and for cases of acne that have resistant to previous treatments with antibiotics or topical medication [1,2].

The mechanism by which isotretinoin act is by normalizing keratinization in sebaceous glands by binding to specific retinoid receptors, modifying gene transcription [1]. Isotretinoin reduce the activity and size of sebaceous gland, keratinization of hair follicles and activity of inflammatory cytokines $[3,4]$ also isotretinoin decrease the number of propionibacterium acnes[5].
Using of this type of vitamin A derivative may cause liver alteration like increase serum level of liver enzymes and lipid changes including increased in triglyceride, total cholesterol and low density lipoprotein (LDL) cholesterol level and decrease the level of high density lipoprotein (HDL)cholesterol [5,6,7].

From ancient time's people in different part of the world, they used herbs and spices as food additives to enhance the taste and flavor of food. Then they learn to use these herbs and spices as a medication to protect or treating them from different disorders and disease. Cumin (scientifically called Cuminum cyminum ) is a second most popular spice in the world especially in India and Mediterranean kitchen. Cumin has been used as anti-inflammatory, diuretic, carminative [8] and antispasmodic, treatment of toothache and epilepsy. Also used as aid for treating dyspepsia, jaundice, diarrhea, flatulent and indigestion[9].Many studies 
reported that cumin used as antioxidant, anti-carcinogenic anthelmintic, anticonvulsant, antimicrobial, hypolipidemic, hepatoprotective and antidiabetic $[10,11,12$, 13]. Phytochemical analysis of cumin shows presence of alkaloid, anthraquinone, coumarin, flavonoid, glycoside, protein, resin, saponin, tannin and steroid [14, 15]. This study try to evaluate the histological damage of liver after treating with the Isotretinoin (same dose descript by dermatology physician for acne treatment) and find out if cumin oil which was antiinflammatory natural herb can protect liver from the damage.

\section{Patients and Methods}

30 male wistar rats weighing 200-250g were used in this study divided into five groups: the first group was control (C group) feeding silage and water, second was treatment group (T1) feeding silage, water and receive $10 \mathrm{mg}$ of Isotretinion. The third group was (T2) feeding silage, water and receives $20 \mathrm{mg}$ of Isotretinion. And last two others were cumin oil groups including group (T3) feeding silage, water and receive $10 \mathrm{mg}$ of Isotretinion plus Cumin oil and (T4) feeding silage, water and receive $20 \mathrm{mg}$ of Isotretinion plus Cumin oil. The study achieved in animal house of college of education- Salahaddin University /Erbil. Animals were maintained in colony rooms with $12 \mathrm{hrs}$. light / dark cycle at $25 \pm 2^{\circ} \mathrm{C}$.

The experiment was accomplished in three weeks. In the end of each week, animals from each group was dissect and liver taken for histological study. Histological diagnosis of all the samples were performed by routine sections and stained with hematoxyline and eosin (H\&E).

\section{Statistical analysis}

Graph Pad Prism 6 used to analyze the data of blood which collected for liver functional enzymes evaluation. Liver functional enzymes include alkaline phosphatase, SGOT, S-GPT, T.S.B, Bilirubin direct and Bilirubin indirect.

\section{Results}

The study which lasted for three weeks, involves 30 male wistar rats which divided into 5 groups: the first group was (C group) which represent negative control group. The second and third groups were control positive treatment groups $\mathrm{T} 1$ and $\mathrm{T} 2$ which received $10 \mathrm{mg}$ and $20 \mathrm{mg}$ of Isotretinion consecutively. The last two others were cumin oil groups which include (T3) that received $10 \mathrm{mg}$ of Isotretinion plus Cumin oil and (T4) that receive $20 \mathrm{mg}$ of Isotretinion plus Cumin oil.

In the end of the study, the liver functional values were studied for each group as it's illustrated in the Table (1). The result shows significant differences between the control groups and treatment (T1) group in the values of alkaline phosphatase as it's appear in the Table (1) and Graph (1). A significant difference also found in the result of serum glutamic oxaloacetic transaminase (S-GOT) between the T2, T3and T4 groups. The T3 group shows the highest significance between the other groups Graph (2). The result of serum glutamate pyruvate transaminase (S-GPT) shows no significant 
differences between the study groups except T3 group which show significant $\mathrm{P}<0.05$ Graph (3).

The data of total serum bile (T.S.B) and indirect bilirubin test shows no significant differences between the study groups as its

Table (1): The value of liver parameters analysis of the rats in the end of the study

\begin{tabular}{|c|c|c|c|c|c|}
\hline Parameters & C group & T1 & $\mathrm{T} 2$ & $\mathrm{~T} 3$ & $\mathrm{~T} 4$ \\
\hline $\begin{array}{c}\text { Alkaline } \\
\text { phosphatase }\end{array}$ & $77 \pm 14.45$ & $83.83 \pm 10.32^{\mathrm{ab}}$ & $74.75 \pm 8.323$ & $55.60 \pm 12.07$ & $76 \pm 10.26$ \\
\hline S- GOT & $121.8 \pm 8.589$ & $98.80 \pm 10.65$ & $100.2 \pm 8.767 *$ & $62.38 \pm 7.717 * *$ & $72.48 \pm 6.480 *$ \\
\hline S- GPT & $29.43 \pm 3.679$ & $22.50 \pm 2.302$ & $22.38 \pm 2.436$ & $16.84 \pm 2.900^{*}$ & $17.86 \pm 2.157$ \\
\hline T.S.B & $0.06500 \pm 0.00866$ & $0.05167 \pm 0.008333$ & $0.07000 \pm 0.01118$ & $0.04600 \pm 0.006782$ & $0.05400 \pm 0.01208$ \\
\hline $\begin{array}{c}\text { Bilirubin } \\
\text { direct }\end{array}$ & $0.01000 \pm 0.000$ & $0.01167 \pm 0.016667^{\mathrm{ab}}$ & $0.01000 \pm 0.000^{\mathrm{b}}$ & $0.02200 \pm 0.005831$ & $0.02800 \pm 0.01114$ \\
\hline $\begin{array}{c}\text { Bilirubin } \\
\text { Indirect }\end{array}$ & $0.05500 \pm 0.00866$ & $0.04000 \pm 0.008165$ & $0.06000 \pm 0.01118$ & $0.02400 \pm 0.007483$ & $0.02600 \pm 0.009274$ \\
\hline
\end{tabular}

*Values are mean \pm SEM of six animals in each group. Data analyzed by one way ANOVA, ${ }^{*} \mathrm{p}<0.05,{ }^{* *} \mathrm{P}<0.01$ and Shapiro-wilk test a (1\%) and Kolmogorov-Smirnov test b (1\%).

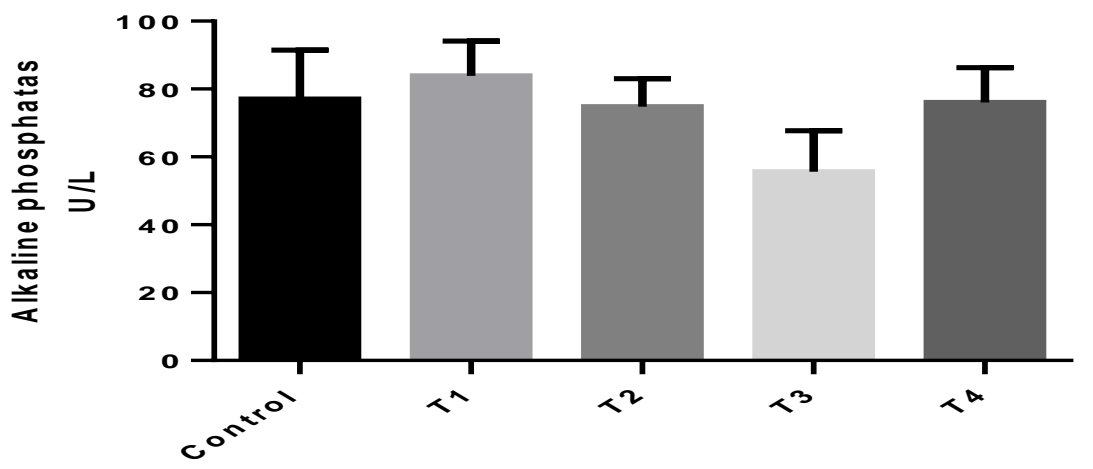

Graph (1): Effect of Isotretinion and cumin oil on the alkaline phosphatase result of rats liver ( $\mathrm{n}=6$ values are mean \pm SEM) 


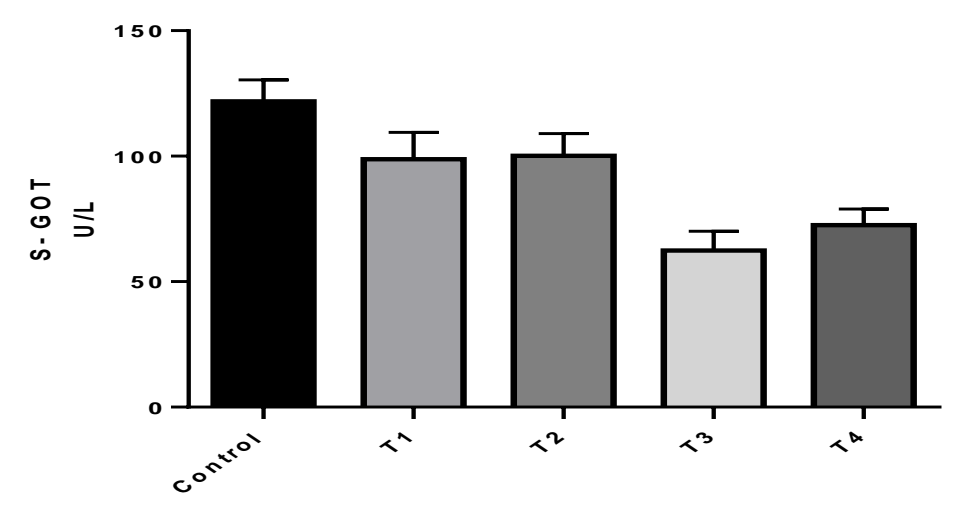

Graph (2): Effect of Isotretinion and cumin oil on the serum glutamic oxaloacetic transaminase (GOT) result of rats liver $(n=6$ are mean \pm SEM)

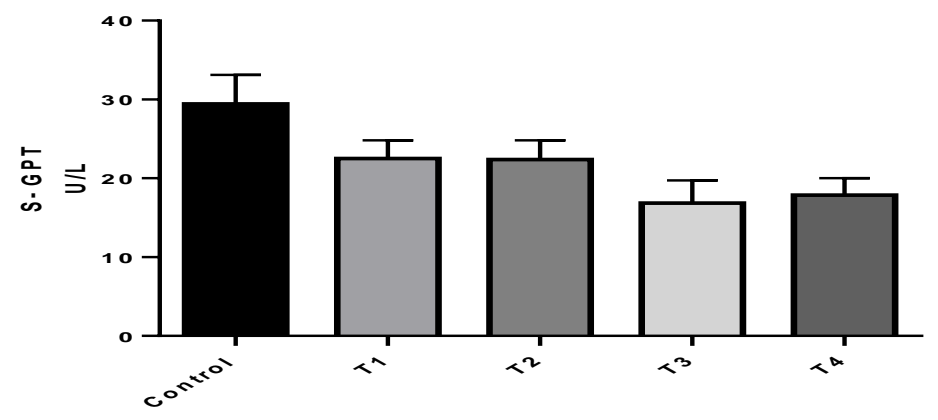

Graph (3): Effect of Isotretinion and cumin oil on the serum glutamate pyruvate transaminase (GPT) result of rats liver $(n=6$ are mean \pm SEM)

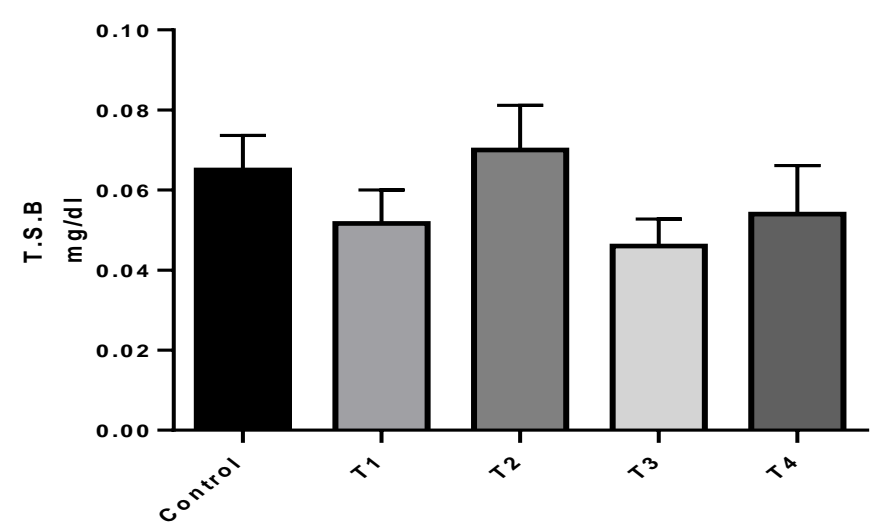

Graph (4): Effect of Isotretinion and cumin oil on the total serum bile (T.S.B) result of rats liver (n=6 are mean \pm SEM) 


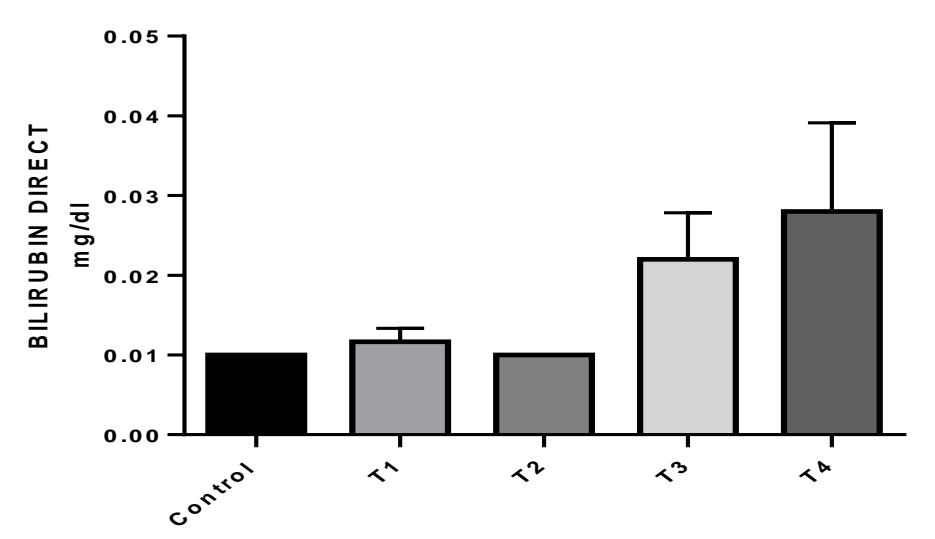

Graph (5): Effect of Isotretinion and cumin oil on the indirect bilirubin result of rats liver ( $\mathrm{n}=6$ are mean \pm SEM)

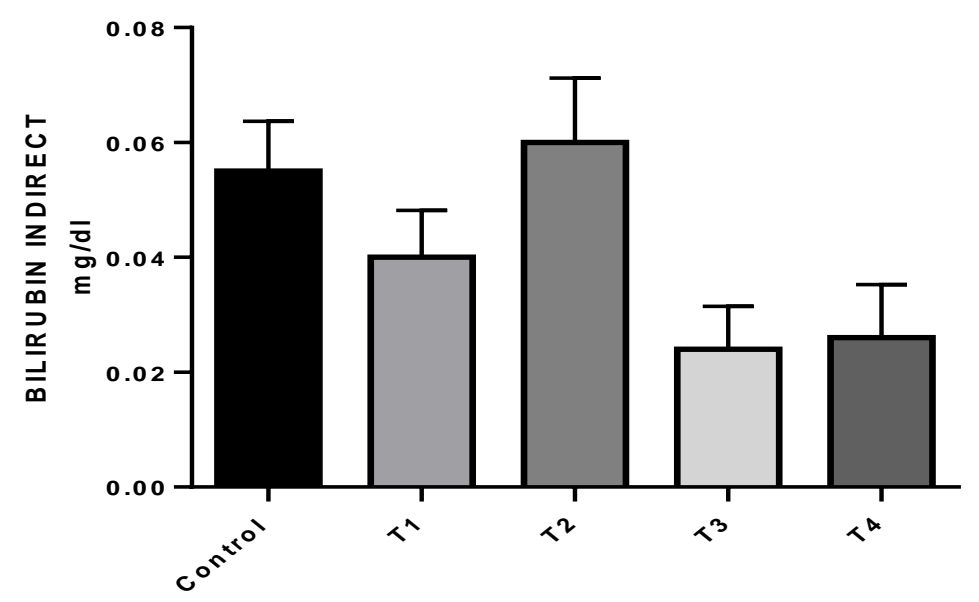

Graph (6):Effect of Isotretinion and cumin oil on the direct bilirubin result of rats liver ( $\mathrm{n}=6$ values are mean \pm SEM)

Microscopic examination of the rat's liver shows variable changes during different time of the study. Isotretinion affect the texture of liver tissue. In the end of the first week of the study, the liver histopathological slide sections of T1 and T2 groups shows infiltration of inflammatory cell around central vein compared to the control group as it appears in Figure 2 and 3 comparing to figure 1. The T2 liver section also shows congestion of sinusoid with vacuolar degeneration in hepatocytes Figure (3). 


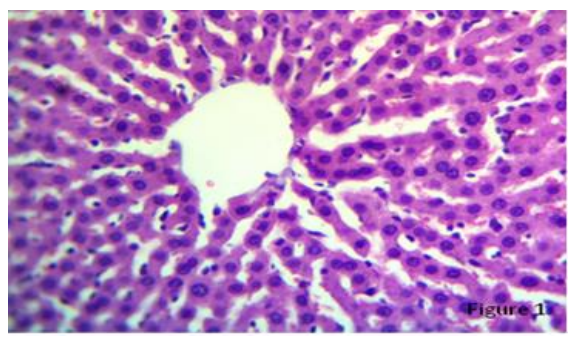

Figure (1): Control group showed normal hepatic tissue arrangement. H\&E 400x

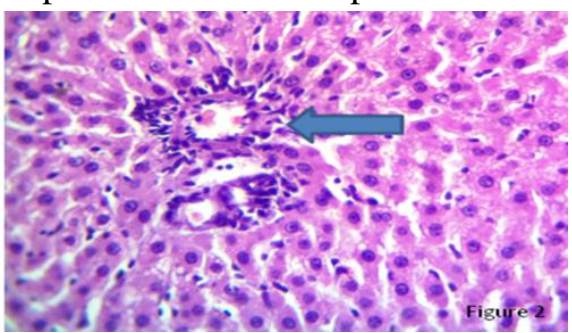

Figure (2): One week liver section of T1 group showed infiltration of inflammatory cell around central vein (blue arrow) H\&E 400x

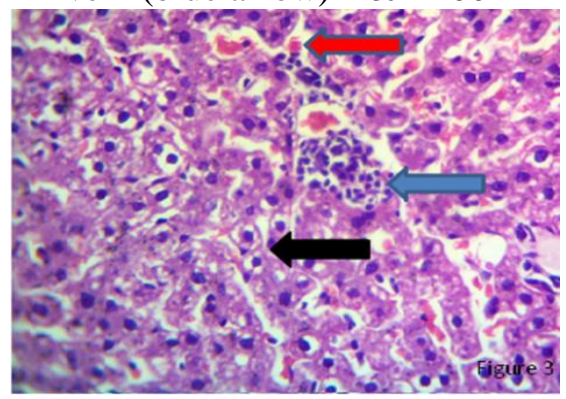

Figure (3): One week liver section of T2 group showed infiltration of inflammatory cell around the central vein (blue arrow), with congestion of sinusoids (red arrow) and vacuolar degeneration in hepatocytes (black arrow) H\&E 400x

The liver histopathological section of $\mathrm{T} 3$ infiltrations of inflammatory cell around group shows dilatation of sinusoid with central vein and few cells showed vacuolar congestion while the T4 shows few generation as its appear in Figure (4) and (5).

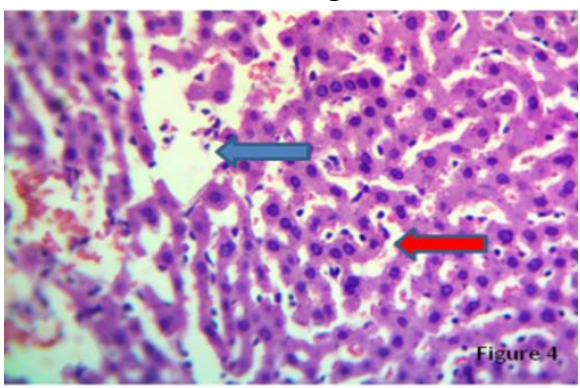

Figure (4): One week liver section of T3 group,showed dilatation of sennosides ( blue arrow), with congestion (red arrow) H\&E. 400x 


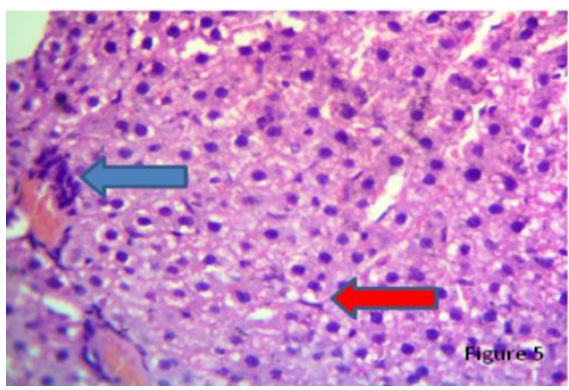

Figure (5): One week liver section of T4 group, showed few infiltrations of inflammatory cell around central vein (blue arrow), with few cells have vacuolar generation (red arrow) H\&E 400x

Significant histopathological changes were observed in liver sections of the treatment study groups in the end of the second week and the changes are highly noticed in the end of third week of the study. The T1 groups liver section shows highly infiltration of inflammatory cell around dilated central vein with vacuolar degeneration in hepatocytes as its appear in Figure (6).

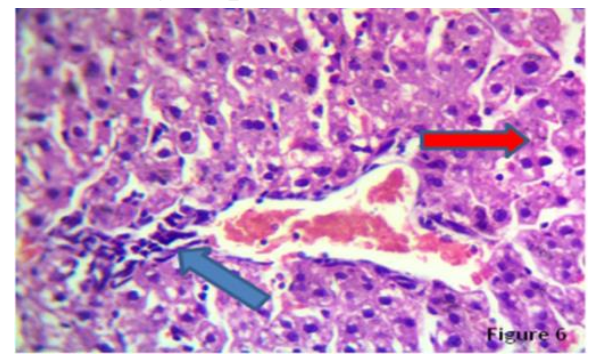

Figure (6): Third week liver section of T1 group, showed infiltration of inflammatory cell around dilated central vein (blue arrow), with vacuolar degeneration in hepatocytes (red arrow) H\&E.

400x

The T2 group which receive $20 \mathrm{mg}$ of also coagulated necrosis in hepatocytes. Isotretinion shows sever infiltration of Figure (7). inflammatory cells around the congested central vein with congestion of sinusoid and

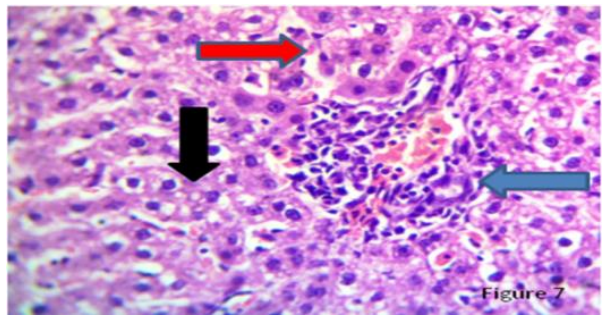

Figure (7): Third week liver section of T2 group, showed sever infiltration of inflammatory cell around congested central vein (blue arrow), with congestion of sinusoids (red arrow) with cogaulative necrosis in hepatocytes (black arrow) H\&E 400x 
In the cumin oil treating groups, the histopathological changes are clearly seen in both second and third weeks of the study. The main features noticed in the last week of the study in the T3 liver section group were dilatation and congestion in central vein beside of cloudy cell swelling in hepatocytes, Figure (8).

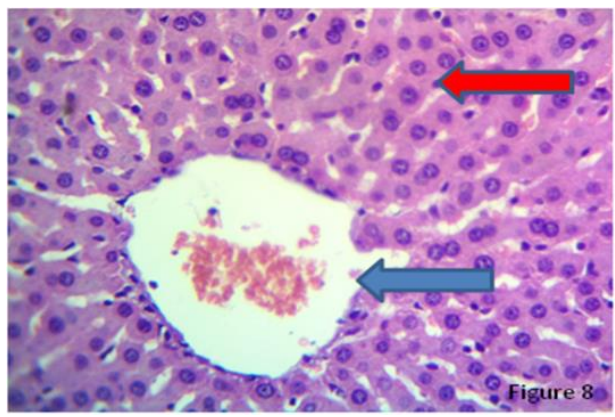

Figure (8): Third week liver section of T3, Showed dilatation and congestion in central vein (arrow), with cloudy cell swelling in hepatocytes (arrow). H\&E. 400x

In the T4 liver section group, the noticed cloudy cell and swelling in other hepatocytes features involve mainly infiltration of with congestion in sinusoids, Figure (9). inflammatory cell around the central vein,

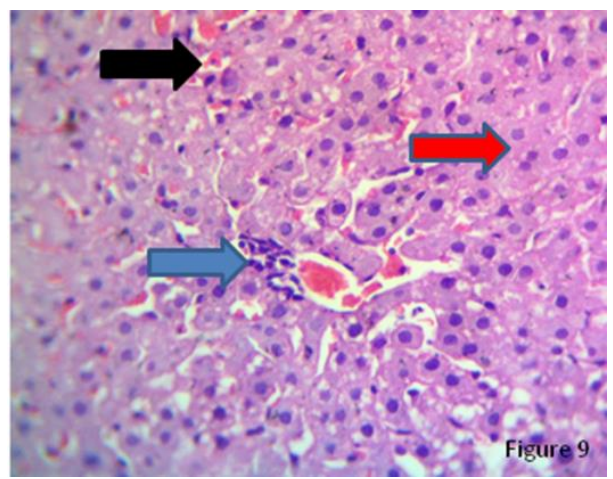

Figure (9): Third week liver section of T4 group, showed few infiltrations of inflammatory cell around central vein (blue arrow), cloudy cell swelling in other hepatocytes (red arrow) with congestion if

\section{Discussion}

Isotretinoin is the most effective treatment for acne, but the long term treatment cause hepatic dysfunction in some patient $[3,16]$. The result of this study clearly shows the effect of isotretinion on the rats liver functional enzymes as it's appear in Table (1) which illustrate the variances between the study groups' data. The variances in the liver functional enzymes during different medicating duration and different dose of isotretinion was confirmed by many of the studies [5, 17], some of these studies indicate that the level of serum GOT and GPT remain under the normal rang or little increase during medication with low dose[18] and that was agree with the result of this study as its 
clearly seen no significant differences in the level of GOT and GPT of the low dose isotretinion $(10 \mathrm{mg})$ treatment group $\mathrm{T} 1$ comparing with control group Table (1), Graph (2) and (3).While a significant differences $(\mathrm{P}<0.05)$ noticed in the level of GOT in the group T2 which treated with high dose of isotretinion (20 mg) as its appear in the table 1and graph2 and that was agree with the study of Okan , Mahmut eata 2014 [5] in this study the cumin oil confirm its protective activity against the effect of isotretinion as its appear in table lin highly significant decrease in level of GOT comparing to the control group level. The cumin oil discerned as anti- inflammatory and antioxidant substance $[9,19]$.

The mechanism by which isotretinion cause serum aminotransferase change is not known , but it may described by direct toxic effect which is appear more frequent with higher dose therapy. The cumin oil has antioxidant activity due to contain mono-terpene alcohol, essential flavor, flavonoids and other polyphenolic molecules 9. The study shows no significant differences between the study groups liver T.S.B. and indirect bilirubin although the cumin oil groups (T3\&T4) shows decreasing in the mean of the TSB and indirect bilirubin as it's noticed in the table 1.in contrast to direct bilirubin which shows significant differences in both low and high dose of isotretinion and that synch with effect of this medication on the hepatotoxicity [20, 21].

The alkaline phosphatase level, usually not affected by the isotretinion dose because of that it's ignored by most of the study. But in this study the low dose of isotretinion cause significant differences ( $\mathrm{T} 1$ group) and the cumin oil efficiently decrease the level of alkaline phosphatase in T3 group Table (1), Graph (1).

The isotretinion cause hepatotoxicity and liver injury[22].The histopathological changes of low and high dose of isotretinion described in this study by infiltration of inflammatory cells, dilation of central vein , vacuolization, degeneration, congestion of sinusoid, necrosis and swelling of hepatocytes. The results of the study showe infiltration of the inflammatory cells in low dose isotretinion treated group (T1) which increased in high dose (T2) comparing to the control group beside congestion of sinusoid and vacuolar degeneration in hepatocytes in the high dose treated group (T2) as its clearly seen in Figure (2) and (3) comparing to figure one .The effect of isotretinion increase with time, the histopathological changes become higher in the second week and sever in the last week of the study as it's clearly noticed in the Figure (6) and (7) and mainly involved infiltration of inflammatory cells beside dilatation of central vein with vacuolar degeneration in hepatocytes in the $\mathrm{T} 1$ group and this histopathological changes become more obvious in $\mathrm{T} 2$ group which shows sever infiltration of inflammatory cells around congested central vein and sinusoid with coagulated necrosis hepatocytes and that's confirm the hepatotoxicity and intense effect of this medication with time [5,21]. The histological examination result of the study 
shows capability of cumin oil in protection from damage effect of isotretinion as its appear in Figure (4) and (5) and aptness of cumin oil in decrease the hepatic alteration changes with long period exposure to the isotretinion Figure (8) and (9). The protective effect of cumin oil was proved by many studies and consider as antiinflammatory substance [19,23] and antioxidant with hepato-protective properties [24].

\section{Conclusions}

The present study conclude that long exposure to isotretinion cause hepatic damage, alteration in the liver texture and affect the functional liver enzyme level specially with high dose. Also the cumin oil is efficient in facing the toxicity effect of isotretinion and reverses the effect.

\section{References}

[1]Andreia Salezze Vieira, Vanessa Beijamini and Ana Carolina Melchiors . The effect of isotretinion on triglycerides and liver aminotransferases.An Bras Dermatol.2012; 87(3): 382-387.

[2]Cooper AJ. Treatment of acne with isotretinion: recommendation based on Australian experience. Astralas J Dermatol.2003; 44:97-105.

[3]Meltem Kumas, Mukaddes Esrefoglu and Eray Metin Guler. Protective effect of silymarin against isotretinion induced liver and kidney injury in mice. Indian journal of experimental biology. 2018; 56:158-163.

[4]Erturan I , Naziroglu M, and Akkaya VB. Isotretinion treatment induce oxidative toxicity in blood of patient with acne vulgaris: a clinical pilot study. Cell Biochem Funct.2012; 30: 554.

[5]Okan Kizilyel, Mahmut Sami Metin , Omer Faruk Elmas, Yasemin Cayir and Akin Aktas. Effect of oral isotretinion on lipid and liver enzymes in acne patients. Cutis.2014; 94:234-238.

[6]Kaymak Yand Ilter N. The result and side effects of systemic isotretinion treatment I 100 patients with acne vulgaris. DermatolNurs.2006; 18:576-580.

[7]Zane LT, Leyden WA, Marqueling Al, manos MM. A population based analysis of laboratory abnormalities during isotretinion therapy for acne vulgaris. Arch Dermatol. 2006; 142:1016-1022.

[8]Mahesh CM, Shivalinge Gowda KP and Ashok Kumar Gupta. Protective action of cuminum cyminum against gentamicin induced nephrotoxicity. Journal of pharmacy research.2010; 3(4):753-757.

[9]Rudra Pratap Singh, Gangadharappa HV and Mruthunjaya K. Cuminum cyminum - A popular spice :An Updated Review. Pharmacogn J. 2017 ;9 (3): 292-301.

[10]Gachkar L, Yadegari D, Razaei MB, Taghizadeh M, Astaneh SA and Rasooli I. Chemical and biological characteristics of Cuminum cyminum and Rosmarinus officinalis oil . Food Chem.2007; 102:898904.

[11]Lacobellis NS, Cantore PL, Capasso F, and Senatore F. Antibacterial activity of Cuminum cyminum and Carum cavi L. essential oils.J Agri Food Chem.2005; 53:5761. 
[12]Sayyah M, Mahboudi A, and Kamalinejad M. Anticonvulsant effect of the fruit essential oil of Cuminum cyminum in mice. Pharmaceutical Biology. 2002; 40: 478-480.

[13]Dhandapani S, Subramanian VR, Rahagopal $S$, and Namasivayam $N$. Hypolipidemic effect of Cuminum cyminum L. on alloxan induced diabetic rats. Pharmacol Res.2002; 46:251-255.

[14]Ali Esmail Al- Snafi. The pharmacological activities of Cuminum cyminum- A review. IOSR Journal of pharmacy. 2016; 6(2):46-65.

[15]Rai N, Yadav S, Verma AK, Tiwari L and Sharma RK. A monographic profile quality specification for a herbal drug and spice of commerce- Cuminum cyminum L. international journal of advanced herbal science and technology .2012; 1(1): 1-12.

[16]Alcalay J, Landau M, and Zucker A. Analysis of laboratory data in acne patients treated with isotretinion: is there really a need to perform routine laboratory tests? J Dermatology Treat.2001; 12: 9-12.

[17]Joseph A, William E, and Gary L. Clinical and laboratory adverse effect associated with long- term, low dose isotretinion: incidence and risk factors. Cancer Epidemiology, Biomarkers and Prevention. 1993; 2: 375-380.

[18]Ahmad H.M. Analysis of clinical efficacy, side effects, and laboratory changes among patients with acne vulgaris receiving single versus twice daily dose of oral isotretinion.Dermatol. Treat. 2015; 28: 151157.
[19]Arun Kumar, Ranjit Kumar, Neeraj Kumar, A. Nath, J.K. Singh, and mohammad Ali. Protective effect of Cuminum cyminum and Coriandrum Sativum on Profenofos Induced Liver Toxicity. International journal of pharmaceutical and biological archives. 2011; 2(5): 1438-1442.

[20]Owen C.E. treating acne with high dose isotretinion. JAMA Dermatol. 2014. 311, 2121.

[21]Deklotz C.M.C., Roby K.D. and Friedlander S.F. Dietary Supplement, Isotretinion and liver toxicity in adolescence: A Retrospective case series. Pediatric. 2017; 140: e 20152940.

[22]Saied N.M and Hamza A.A. Selenium ameliorate isotretinion -induced liver injury and dyslipidemia via antioxidant effect in rats. Toxicol. Mech. Methods. 2014; 24: 433437.

[23]Salah. A Sheweita, Lobna S. El-Hosseiny and Munther A.Nashashibi. Protective effect of essential oils as natural antioxidant against hepatotoxicity induced by cyclophosphamide in mice. PLOS One.2016; DOl: 10.137: 0165667.

[24]Nadeem $M$ and Riaz A. Cumin ( Cuminum cyminum) as a potential source of antioxidants. Pak J Food Sci.2012; 22: 101107. 